predictor of outcome. (Tekgul H, Gauvreau K, Soul J, et al. The current etiologic profile and neurodevelopmental outcome of seizures in term newborn infants. Pediatrics April 2006;117:1270-1280). (Respond: Andre J du Plessis MD, Fegan 11, Department of Neurology, Children's Hospital, 300 Longwood Ave, Boston, MA 02115).

COMMENT. Mortality is relatively low but long-term neurodevelopmental outcome is poor in $28 \%$ of infants with a history of neonatal seizures. Global cerebral hypoxicischemia is the most frequent cause of neonatal seizures and a strong predictor of poor longterm outcome. The effect of neonatal seizures on the developing brain is controversial, some finding them harmful (Wasterlain CG. Epilepsia 38:728-734) and some, harmless (Camfield PR. Epilepsia 1997;38:735-737). Studies in experimental animals have shown that immature rats less than 20 days old respond to electroshock with hyperkinesias, breast stroke swimming movements, tremors, catatonia, and clonic movements, and a major tonic-clonic seizure with post-ictal depression could not be induced until the animal was older. (Millichap JG. Proc Soc Exper Biol \& Med 1957;96:125-129). Neonatal seizures in infants (Volpe JJ, 1977) are described as subtle and include the swimming movements resembling those seen in animals. The absence of generalized tonic-clonic seizures in the neonate reflects the lack of cortical organization required to propagate the electrical discharge (Aicardi J, 1986). These findings support those of Camfield and others who discount adverse effects of neonatal seizures per se, attributing the poor prognosis to the cause, especially cerebral hypoxiaischemia. Subtle and focal post-ictal cerebral ischemia affecting subcortical and limbic regions cannot be ruled out.

Relation of pregnancy and neonatal factors to development of childhood epilepsy. Prenatal factors contributed to the subsequent development of childhood epilepsy in a study in Nova Scotia, Canada (Whitehead E, et al. Pediatrics April 2006;117:12981306). Risk factors for epilepsy included eclampsia, CNS anomalies, placenta abruptio, infection in pregnancy, and unmarried mother. Other factors associated with increased risk of epilepsy were neonatal seizures, neonatal metabolic disorders, low birth weight, and small for gestational age.

\title{
PROLONGED FEBRILE SEIZURES AND TEMPORAL LOBE EPILEPSY
}

The presence of spontaneous limbic seizures using chronic video monitoring with concurrent hippocampal and cortical EEGs, in adult rats (starting at 3 months of age) that had sustained experimental febrile seizures (FS) on postnatal day 10 was compared to hyperthermic control rats whose FS had been controlled, in a study at University of California, Irvine, CA, and University of Pennsylvania, Philadelphia. EEGs were normal in hyperthermic control rats, and none developed spontaneous seizures. In rats subjected to prolonged early-life FS, spontaneous electroclinical seizures were recorded in 6 of 17 (35.2\%). The seizures, sudden freezing and typical limb automatisms, were associated with polyspike/sharp waves with increasing amplitude and slowing on EEG. Interictal epileptiform discharges were recorded in $15(88.2 \%)$ of the experimental seizure rats and in none of the controls. A diminished amplitude of cortical EEG preceding the hippocampal seizure suggests involvement of normal cortical neurons. This represents a model for studying the relation between FS and human temporal lobe epilepsy. (Dube C, Richichi C, 
Bender RA, et al. Temporal lobe epilepsy after experimental prolonged febrile seizures: prospective analysis. Brain April 2006;129:911-922). (Respond: Tallie Z Baram MD PhD, Department of Anatomy and Neurobiology and Department of Pediatrics, University of California at Irvine, ZOT 4475, Irvine, CA 92697).

COMMENT. These laboratory studies support the theory that prolonged infantile FS may predispose to later- onset temporal lobe epilepsy (TLE) (Annegers JF, et al. N Engl J Med 1987;316:493-498). The duration of the FS has been shown to correlate with prognosis and the later development of epilepsy and epileptiform EEGs (Millichap JG et al. Neurology $1960 ; 10: 643-653)$. Epilepsy developed in $30 \%$ of patients with prolonged FS ( $>20 \mathrm{~min}$ ) and in only $5 \%$ of those with short FS $(<20 \mathrm{~min})$. The incidence of EEG abnormalities at followup in patients with long and short FS was $36 \%$ and $10 \%$, respectively. In a more recent study, the mean duration of the FS was $100+/-133$ min in patients who later developed TLE and 9+/-19 min in those without TLE at prolonged follow-up (mean 32 years) (Maher J, McLachlan RS. Brain 1995;118:1521-1528). The prevention of febrile seizures, especially the complex type, requires more effective therapeutic intervention.

\section{ABSENCE EPILEPSY AND MOYAMOYA DISEASE}

The case of a 6-year-old girl with typical absence epilepsy associated with moyamoya disease (MMD) is reported from the Department of Neurosurgery, Kyoto University Graduate School of Medicine, Japan. The child had suffered from recurrent syncope, approximately five times a day for one year, prior to the onset of absence. At 4 years of age she developed episodes of bilateral weakness of her legs, induced by hyperventilation. An EEG revealed a typical bilateral synchronous $3-\mathrm{Hz}$ spike-and-wave discharge induced by hyperventilation. With high-dose sodium valproate (1800 mg daily) seizures increased in frequency to 10 times daily. An MRI showed a small infarction in the left frontal lobe, and cerebral angiography revealed stenoocclusion of terminal portions of both internal carotid arteries and moyamoya vessels at the base of the brain. Antiplatelet therapy with aspirin was started, and decreased regional cerebral blood flow on SPECT and decreased cerebrovascular reserve were demonstrated in bilateral frontal lobes, presurgically. Following bilateral superficial temporal artery-middle cerebral artery anastomoses, the patient responded to treatment with sodium valproate and aspirin, and she had been free of seizures and transient ischemic attacks for more than 2 years at follow-up. An EEG with hyperventilation after withdrawal of medication showed no bursts of spike-and-wave. This is the first reported case of absence epilepsy associated with MMD. (Kikuta K-I, Takagi Y, Arakawa Y, et al. Absence epilepsy associated with moyamoya disease. Case report. J Neurosurg (4 Suppl Pediatrics) April 2006;104:265-268). (Reprints: Ken-ichiro Kikuta MD PhD, Department of Neurosurgery, Kyoto University Graduate School of Medicine, 54 Kawaharacho, Shogoin, Sakyo-ku, Kyoto 606-8507, Japan).

COMMENT. Moyamoya disease should be considered in the diagnosis of refractory absence epilepsy in Japan, but the prevalence is extremely low. In this case, the preceding syncopal episodes were explained by transient ischemic attacks, and ischemia in the frontal lobes enhanced by hyperventilation was probably related to the occurrence of absence seizures. A possible genetic factor was omitted from the report. 